\title{
ARTICLE \\ Effect of Sleep Quality on Mental Health during COVID- 19 Lockdown
}

\author{
Aastha Jain* \\ Psychologist at Cankids Kidscan (NGO), Masters in Psychology (NET Qualified), India
}

\section{ARTICLE INFO}

Article history

Received: 29 April 2021

Accepted: 31 May 2021

Published Online: 10 July 2021

Keywords:

Sleep quality

Mental health

Lockdown

\begin{abstract}
The study was done with the aim of understanding the "Effect of Sleep Quality on Mental Health of Adults during COVID-19 Lockdown". 136 adults aged between 18 to 32 years were selected through snowball sampling from social media platforms for being a part of the study sample. The Sleep Condition Indicator (Espie, 2014) and General Health Questionnaire (GHQ-12) (Goldberg and Williams, 1988) were used to measure their sleep quality and mental health. It was hypothesized that the sleep quality will have a significant effect on the mental health of adults. It was found out that sleep quality is a significant predictor of mental health. The researcher stated that clinicians and psychologists should investigate about the sleeping patterns and quality of sleep in patients reporting mental health issues, and screen sleeping disorders regularly in population to avoid development of mental health disorders.
\end{abstract}

the circadian rhythm of the body, and hence affecting the sleep and mood of the people ${ }^{[6]}$. Hence, it is very important to recognise and treat sleep related issues in people, specially during the times of stress, like lockdown and the outbreak of a deadly virus ${ }^{[3]}$ as sleep is linked and considered a crucial element of psychological health, and has also been linked to psychopathology ${ }^{[7]}$.

\section{Review of Literature}

Shamshri, Moshki and Mogharab (2014) ${ }^{[8]}$ did a study "The survey of sleep quality and its relationship to mental health of hospital nurses" in Gonadan and Birjan cities. They studied 260 nurses. Pittsburg Sleep Quality Index and General Health Questionnaire were used for measuring the status of their sleep quality and mental health respectively. It was found out that $56.2 \%$ nurses had poor mental health and $43.8 \%$ nurses had good mental health. The sleep quality was poor/ unfavourable in $69.2 \%$ nurses and only $30.8 \%$ nurses reported good sleep quality.

*Corresponding Author:

Aastha Jain,

Psychologist at Cankids Kidscan (NGO), Masters in Psychology (NET Qualified), India;

Email: aastha_jain08@yahoo.com 
A statistically significant relationship was concluded by computing chi-square test.

Seun-Fadipe and Mosaku (2017) ${ }^{[9]}$ did a study to evaluate "Sleep quality and psychological distress among undergraduate students of a Nigerian university". 505 participants were selected by multistage sampling method. Pittsburg Sleep Quality Index, General Health Questionnaire, Hospital-Anxiety Depression Scale and socio- demographic questionnaire were used to assess the participants. $50.1 \%$ students reported having poor sleep quality, out of which $5.7 \%$ used to take sleep medications once or twice a week. $24.4 \%$ students had psychological distress. Also, year of study was significantly related to poor sleep quality. Also, psychological distress and depressive and anxiety symptoms were associated with poor sleep quality. It was concluded that symptoms of anxiety and psychological distress were significantly predictors of poor sleep quality. They also concluded that a significant relationship between sleep quality and psychological distress further advocates the importance of inculcating habits which improve sleep quality and mental health among undergraduate students.

Coiro et al., (2021) ${ }^{[10]}$ studied the "Sleep quality and COVID- 19 related stress in relation to mental health symptoms among Israeli and U.S. adults". They collected cross-sectional data through online surveys, from 2541 community adults of Israel and USA, aged between 1870 years. Measures related to COVID- 19 related stress, sleep quality, anxiety, depression and adjustment disorder related symptoms. The results revealed that symptoms of depression, anxiety and adjustment disorder were prevalent along with poor sleep quality. Also, sleep disturbances mediated the relationship between stress related to COVID- 19 and symptoms of anxiety and depression.

Freeman, Sheaves, Waite, Harvey and Harrison (2020) ${ }^{[11]}$ did a review to investigate whether disrupted sleep was a contributing factor in the occurrence of mental health disorders. They concluded that insomnia and mental health conditions have some common causal factors, and along with this, they both share a bidirectional relationship with each other also. They stated that treating sleep disorders in early stages might prevent the onset of many psychiatric disorders. They also recommended that insomnia should be regularly assessed, that sleeping issues and difficulties should be treated as a problem in their own right, and at the same time should be recognized as a means to reduce the occurrence of other mental health issues, along with expansion for evidence based treatment for sleeping disorders in mental health services should be made easily accessible.
Yun (2016) ${ }^{[12]}$ did a study to investigate the relationship between sleep quality and mental health of adolescents and identify the factors related to mental health. 285 middle school students were studied for this purpose. The data were analyzed using t-test, ANOVA, Pearson's correlation coefficient and multiple linear regression with SPSS ver. 21. The mean score of sleep quality of adolescents was 4.20 and the mean score of mental health was 13.67. It was seen that the adolescents who reported having poor sleep had high score on the measure of mental health than their counterparts who had better sleep quality. Mental health had a positive relationship with sleep quality and stress. Also, it was concluded that sleep quality and stress were significant factors which affect mental health and they explained 59\% variation in mental health. It was concluded that effective intervention programs for improving the quality of sleep of adolescents should be implemented for enhancing their mental health.

Ghrouz et al. (2019) ${ }^{[13]}$ did a study to examine the "Physical activity and sleep quality in relation to mental health among college students". They selected a sample of 617 college students from Indian colleges, aged between 18 to 30 years. They were assessed using three questionnaires- the Hospital Anxiety and Depression Scale, the International Physical Activity QuestionnaireShort Form, and the Pittsburgh Sleep Quality Index. They found out that the prevalence of anxiety was more than that of depression, and $51 \%$ of the participants reported that they have low physical activity levels as well as poor sleep quality. They also found out that poor sleep quality was related with depression and anxiety.

\section{Objective}

The objective of the study is to investigate the effect of sleep quality on mental health during the COVID-19 lockdown.

\section{Hypothesis}

H1: There will be a significant effect of sleep quality on the mental health of adults.

\section{Methodology}

\subsection{Sample}

The sample was collected through snowball sampling on online social media platforms. The sample size was 136 people (48 males; 88 females), aged between 18 to 32 years. Further details about their marital and employment status were also recorded. Out of the total sample 60 were employed and 64 were unemployed, and 108 were 
unmarried and 28 were married.

\subsection{Tools}

The data were collected using the The Sleep Condition Indicator (Espie, 2014) ${ }^{[14]}$ and General Health Questionnaire (GHQ- 12) (Goldberg, 1988) ${ }^{[15]}$.

\subsubsection{The Sleep Condition Indicator (Espie, 2014)}

The scale is an 8 item questionnaire, which was developed to screen sleeping disorders, based on the criteria given by DSM-5. Against every item, 5 options are given, and the subject is asked to read every item and mark the response accordingly. Every answer is scored on a scale of 0 to 4 .

The scoring is done by totalling all the response scores. The scores can range between 0 and 32. A higher score depicts a better sleep quality and less symptoms of insomnia and other sleep related issues.

The tool has robust internal consistency $(\alpha \geq 0.86)$ and showed convergent validity with the Pittsburgh Sleep Quality Index and Insomnia Severity Index ( $r=0.90)$.

\subsubsection{General Health Questionnaire (GHQ- 12) (Goldberg, 1988)}

This questionnaire is used widely for assessing the mental health of patients and general public. GHQ originally had 60 questions, but it has several versions. The one used for this study is GHQ-12. It has 12 items, which are based on a 4 point likert scale. The respondents are asked to read the items and mark the most suitable response, which is then scored on a scale of 0 to 3 .

The scores at the end are added to derive an overall total score, which can range between 0 to 36 . A higher score depicts a poorer mental health and worse conditions.

The reliability of the tool is 0.90 , and the external validity of the test is 0.58 (with subjective well-being inventory).

\subsection{Statistical Analysis}

The data were then analysed with the help of SPSS, and linear regression was computed to test the hypothesis.

\subsection{Results and Interpretation}

In this study, the dependent variable is mental health, and the independent variable is sleep quality. The objective of this study was to investigate the effect of sleep quality on the mental health during the COVID-19 lockdown in India.

It was hypothesized that there will be a significant effect of sleep quality on the mental health of adults, during COVID-19 lockdown, and the result is shown below.

Table 1. Regression analysis summary for predicting mental health of adults during COVID-19 lockdown.

\begin{tabular}{|c|c|c|c|c|c|c|}
\hline \multicolumn{7}{|c|}{ Coefficients $\mathrm{s}^{\mathrm{a}}$} \\
\hline & \multirow{2}{*}{$\begin{array}{l}\text { Model } \\
\text { B }\end{array}$} & \multicolumn{2}{|c|}{$\begin{array}{l}\text { Unstandardized } \\
\text { Coefficients }\end{array}$} & \multirow[t]{2}{*}{$\begin{array}{c}\text { Standardized } \\
\text { Coefficients } \\
\end{array}$} & \multirow{2}{*}{$\mathrm{T}$} & \multirow{2}{*}{ Sig. } \\
\hline & & Std. Error & Beta & & & \\
\hline \multirow[b]{2}{*}{1} & (Constant) & 30.774 & 1.634 & & 18.831 & .000 \\
\hline & $\begin{array}{l}\text { Sleep } \\
\text { Quality }\end{array}$ & -.334 & .101 & -.276 & -3.319 & .001 \\
\hline
\end{tabular}

$\mathrm{R} 2$ adjusted $=0.076$

A simple liner regression was calculated to predict the effect of sleep quality on mental health of adults during COVID-19 lockdown. A significant regression equation was found $(\mathrm{F}(1,134)=11.01, \mathrm{p}<0.01)$, with an $\mathrm{R}^{2}$ of 0.076 . Mental health is increased by $7.6 \%$ for increase in each unit in sleep quality. The correlation coefficient was computed to be -0.276 . A higher score on General health Questionnaire indicates worse condition, and a higher score on sleep quality indicates better sleep quality, so a negative relationship here depicts that better sleep quality is related with better general (mental) health.

The value of regression analysis is statistically significant, and hence it can be stated that the effect of sleep quality on mental health of adults in India, during COVID-19 lockdown is significant. The hypothesis has thus been accepted.

\section{Discussion}

The results of the study reveal that there is a direct causal relationship between sleep quality and mental health of adults, and thus the hypothesis has been accepted. It can also be stated that sleep quality directly affects mental health of adults, and it did so during the lockdown that was imposed by the government during the outbreak of COVID-19 virus. A poor sleep quality is directly related to poor mental health and vice versa. So if people who had disturbed sleeping patterns and had poor sleep quality, reported that their mental health was also worse in contrast to people/adults who had better sleep quality.

Hence, for improving the overall mental health of people, especially during stressful times, like lockdown, clinicians should focus on the steps for improvement of sleep quality.

Some factors which contributed to poor sleep quality in some adult groups ${ }^{[3]}$ during lockdown were irregular 
working hours, disruption of sleep-wake cycle, lack of physical activity, increased screen time, stress related to finances, job insecurity, underlying health conditions and COVID anxiety. Poor sleep quality is a significant predictor of deterioration in mental health, and so it can be concluded that people suffered with disturbed mental health during the tough times. And clinicians and psychologists can thus, investigate their sleep quality and patterns to help them deal with their mental health issues more effectively. They can provide their patients with practices and techniques to enhance their sleep quality, like sleep hygiene and relaxation techniques.

In clinical setups also, when patients report poor mental health and other mental health related issues, clinicians can look for sleep related issues to have a better understanding. And, patients who report poor sleep, can also be screened for potential mental health deterioration.

Similar results have been quoted by other researchers. In a review done by Freeman, Sheaves, Waite, Harvey and Harrison (2020) ${ }^{[10]}$, it was stated that insomnia and mental health conditions share a bidirectional relationship. They recommended that insomnia and other sleep issues should be assessed regularly in case of mental health disorder.

The results of this study are important because it provides a pathway for understanding the importance of sleep and related issues in psychiatric conditions, and poor mental health of individuals. The results help us to realise the importance of exploring the sleep quality of people who report other mental health related conditions, like stress, depressive symptoms, anxiety, emotional disturbance etc., and how identifying sleep difficulties in preliminary stages can prevent occurrence of mental health disorders. This study contributes to the literature which establishes a cause and effect relationship between poor sleep quality and mental health issues, and provides an evidence to clinicians for the same.

The researchers who wish to pursue and explore this relationship further, may also try to investigate the existence of gender differences, if any, and how mental health also contributes to disturbed sleeping patterns. Also, they may explore this further in non-stressful times. This study was done during COVID-19 lockdown in India, and so the results may have been influenced by other factors that played a role in times of widespread stress, so replicating the results in times when everything is regular on national/community level, may strengthen the evidence even further. Also, this relationship may be tested for different age groups, along with comprehending what else contributes to poor mental health apart from poor sleep quality.

\section{Conclusions}

The study was done with the aim of understanding the "effect of sleep quality on mental health of adults during COVID-19 lockdown". 136 adults aged between 18 to 32 years were selected through snowball sampling from social media platforms for being a part of the study sample. The Sleep Condition Indicator (Espie, 2014) and General Health Questionnaire (GHQ-12) (Goldberg and Williams, 1988) were used to measure their sleep quality and mental health. It was found out that sleep quality is a significant predictor of mental health.

Hence the study concludes that sleep quality affects the mental health of the adults significantly, and it was a significant predictor of adults' mental health during the COVID-19 lockdown.

The researcher, based on the results, suggests the clinician to screen for insomnia and other sleep issues in early stages of treatment, to prevent the patients from developing mental health disorders later, and also screen for sleep related disorders and issues in patients who report poor mental health.

\section{References}

[1] Gupta, R., Grover, S., Basu, A., Krishnan, V., Tripathi, A., Subramanyam, A., ... \& Avasthi, A. (2020). Changes in sleep pattern and sleep quality during COVID- 19 lockdown. Indian Journal of Psychiatry, 62(4), 370.

[2] Brooks, S. K., Webster, R. K., Smith, L. E., Woodland, L., Wessely, S., Greenberg, N., et al. (2020). The psychological impact of quarantine and how to reduce it: rapid review of the evidence. Lancet 395, 912-920.

[3] Jain A., (2020). Quality of sleep during the coronavirus (COVID- 19) outbreak. International Journal of Indian Psychology, 8(3), 1826-1831.

[4] Grima, N. A., Bei, B., \& Mansfield, D. (2019). Insomnia theory and assessment. Australian journal of general practice, 48(4), 193.

[5] Martire, V. L., Caruso, D., Palagini, L., Zoccoli, G., \& Bastianini, S. (2019). Stress \& sleep: a relationship lasting a lifetime. Neuroscience \& Biobehavioral Reviews.

[6] McClung, C. A. (2013). How might circadian rhythms control mood? Let me count the ways... Biological psychiatry, 74(4), 242-249.

[7] Hertenstein, E., Feige, B., Gmeiner, T., Kienzler, C., Spiegelhalder, K., Johann, A., ... \& Baglioni, C. (2019). Insomnia as a predictor of mental disorders: a systematic review and meta-analysis. Sleep medicine 
reviews, 43, 96-105.

[8] Shamshri, M., Moshki, M., \& Mogharab, M. (2014). The survey of sleep quality and its relationship to mental health of hospital nurses. Iran Occupational Health Journal, 11.

[9] Seun-Fadipe, C. T., \& Mosaku, K. S. (2017). Sleep quality and psychological distress among undergraduate students of a Nigerian university. Sleep Health, 3(3), 190-194.

[10] Coiro, M. J., Asraf, K., Tzischinsky, O., Hadar-Shoval, D., Tannous-Haddad, L., \& Wolfson, A. R. (2021). Sleep quality and COVID- 19-related stress in relation to mental health symptoms among Israeli and US adults. Sleep Health, 7(2), 127-133.

[11] Freeman, D., Sheaves, B., Waite, F., Harvey, A. G., $\&$ Harrison, P. J. (2020). Sleep disturbance and psy- chiatric disorders. The Lancet Psychiatry, 7(7), 628637.

[12] Yun, H. J. (2016). Effect of stress and sleep quality on mental health of adolescents. Journal of the Korean Society of School Health, 29(2), 98-106.

[13] Ghrouz, A. K., Noohu, M. M., Manzar, M. D., Spence, D. W., BaHammam, A. S., \& Pandi-Perumal, S. R. (2019). Physical activity and sleep quality in relation to mental health among college students. Sleep and Breathing, 23(2), 627-634.

[14] Espie, C. A., Kyle, S. D., Hames, P., Gardani, M., Fleming, L., \& Cape, J. (2004). The Sleep Condition Indicator: a clinical screening tool to evaluate insomnia disorder. BMJ open, 4(3).

[15] Goldberg, D. P. (1988). User's guide to the General Health Questionnaire. Windsor. 